UDC: 519.633 .2

\title{
Overset grids approach for topography modeling in elastic-wave modeling using the grid-characteristic method
}

\author{
N. I. Khokhlov ${ }^{a}$, V. O. Stetsyuk ${ }^{b}$, I. A. Mitskovets ${ }^{c}$ \\ Moscow Institute of Physics and Technology, \\ 1A Kerchenskaya st., Moscow, 117303, Russia \\ E-mail: ${ }^{\mathrm{a}} \mathrm{k}$ _h@inbox.ru, ${ }^{\mathrm{b}}$ stetsyuk@phystech.edu, ${ }^{\mathrm{c}}$ mitkovets@phystech.edu
}

\begin{abstract}
While modeling seismic wave propagation, it is important to take into account nontrivial topography, as this topography causes multiple complex phenomena, such as diffraction at rough surfaces, complex propagation of Rayleigh waves, and side effects caused by wave interference. The primary goal of this research is to construct a method that implements the free surface on topography, utilizing an overset curved grid for characterization, while keeping the main grid structured rectangular. For a combination of the regular and curve-linear grid, the workability of the grid characteristics method using overset grids (also known as the Chimera grid approach) is analyzed. One of the benefits of this approach is computational complexity reduction, caused by the fact that simulation in a regular, homogeneous physical area using a sparse regular rectangle grid is simpler. The simplification of the mesh building mechanism (one grid is regular, and the other can be automatically built using surface data) is a side effect. Despite its simplicity, the method we propose allows us to increase the digitalization of fractured regions and minimize the Courant number. This paper contains various comparisons of modeling results produced by the proposed method-based solver, and results produced by the well-known solver specfem $2 \mathrm{~d}$, as well as previous modeling results for the same problems. The drawback of the method is that an interpolation error can worsen an overall model accuracy and reduce the computational schema order. Some countermeasures against it are described. For this paper, only two-dimensional models are analyzed. However, the method we propose can be applied to the three-dimensional problems with minimal adaptation required.
\end{abstract}

Keywords: overset grid, GCM, seismic wave, interpolation

Citation: Computer Research and Modeling, 2019, vol. 11, no. 6, pp. 1049-1059.

This work was supported by the Russian Foundation for Basic Research, project no. 18-31-20041 mol_a_ved. 


\section{Introduction}

The problem of simulation of seismic wave propagation is fundamental. Many realistic seismology problems make us consider nontrivial topology, for instance, it is absolutely essential in cases where we need to simulate the process of wave propagation in areas located not far (a few hundred meters) from Earth's surface, soil fluctuations or cracks in soil. All these features have a significant impact on the propagation of seismic waves. The heterogeneity of the topography may cause such an unexpected phenomenon as seismic wave diffraction and interference [Tarrass et al., 2011]. Due to the evolution of computer science and hardware development nowadays we are able to use various numeric methods to solve such type of problems.

Various approaches exist for the calculation of seismic wave propagation, its finite-element, finite-difference, finite-volume, spectral and pseudo-spectral methods [Virieux et al., 2011; Zhang et al., 2012b].

Finite-element methods suitable for seismic wave propagation include the spectral-element method [Komatitsch, Tromp, 1999; Dortdivanlioglu et al., 2018] and the discontinuous Galerkin method [Etienne et al., 2010]. These approaches are the most appropriate for modeling in the case of nontrivial topography due to their ability to use discretized meshes, accurately describing curves of surface [Gao et al., 2015; Tarrass et al., 2011], such a quality takes its price, high computational complexity and complex unstructured grid generation. The latest research shows the ability of the finite element approach to simulate even absolutely absorbing boundary conditions [Liu et al., 2014; Meng, $\mathrm{Fu}, 2017]$.

Finite-difference is one of the leading methods for seismic modeling due to its simplicity and accuracy in the presence of flat describing topology. It reaches an excellent resolution near the freesurface boundary [Gottschammer, 2001; Kristek et al., 2002]. But the ordinary FD method is only valid for the planar surface. A few methods exist to account for complex topology using FD, the first one applies staggered grids [Zeng et al., 2012a; Cao, Chen, 2018] or partly staggered grids [Jianfeng, 1997; Cruz-Atienza, Virieux, 2004] with vacuum nodes, which leads to staircase-like topography of solution. In simple vacuum formulation for the FD method, in which the physical parameters of the grid nodes above the free surface(vacuum nodes) are set to zero, it is suitable only for second-order spatial operators [Graves, 1996]. In [Cao, Chen, 2018] a parameter-modified method was presented which allows eliminating diffraction caused by the staircase grid with only 15 points per minimum wavelength.

Also, there are methods based on structured grids that match the topography, where those are grids reduced to the form of rectangle grids by coordinate transformation, and the finite-difference approach with free-surface boundary conditions applies, but this nonorthogonality in this approach reduces the accuracy of the waveform simulation [Gao et al., 2015; Petersson, Sjögreen, 2015; Pérez et al., 2016]. To eliminate this disadvantage, a summation-by-parts (SBP) finite-difference method was used to simulate seismic wave propagation in the presence of fluctuating interfaces [Peng et al., 2018], this method requires that no significant restrictions be imposed on the grid, and allows accuracy degradation to be overcome.

Some publications describe interesting methods that represent FE-FD approaches, trying to take advantage of FD as a volumetric method and keep FE as a boundary method to achieve its accuracy near free-surface [Ma, 2004].

After our comparison of SEM and DGM approaches for seismic wave propagation problems based on [Peter et al., 2011; Biryukov et al., 2016], we may draw a conclusion on their comparable accuracy with comparable runtime per one degree of freedom. A comparison of FD, FE, SE, and DG methods for the same problem statement is well studied in [Jianfeng, 1997]. Moreover, similar research of such methods as DGM, GCM on unstructured meshes and shock-capturing GCM on structured 
meshes are described in [Biryukov et al., 2016]. In [Petrov et al., 2017], GCM have been applied on embedded hierarchical grids to investigate the destruction in complex heterogeneous structures caused by seismic waves propagating from an earthquake hypocenter. We want to represent the meaning of a similar approach. In [Favorskaya et al., 2018; Stognii, Khokhlov, 2019], the application of the GCM technique was shown and the recent developments in the GCM technique for solving the elastic wave equation were presented, in particular, nonuniform grids with different cell sizes in different areas and nonuniform nodes were used.

In this paper, we investigate the workability of the GCM approach using overset grids (also known as the Chimera grid approach) in a combination of regular rectangular and curvilinear grids. The same study with rectangular rotated grids was presented in "Modeling of Fractures Using the Chimera Grid Approach" [Ruzhanskaya, Khokhlov, 2018]. Figures 1, 2 and 3 illustrate the most commonly used grid types along with the proposed grid layout for the same topology. We will show the allocation of cracks and surface by overlaying a curved grid to characterize fractures. Furthermore, such a mechanism allows us to retrench computational complexity by simulating wave propagation in a regular, homogeneous physical area using a sparse regular rectangle grid, so we can increase the digitalization of fractured regions and minimize the Courant number. As a bonus, a mechanism of the mesh building is simplified.

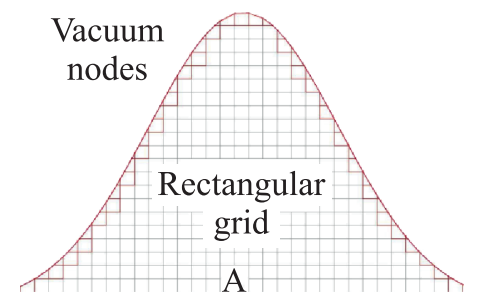

Figure 1. Cartesian cut cell. Cells near-surface are beveled by the red line

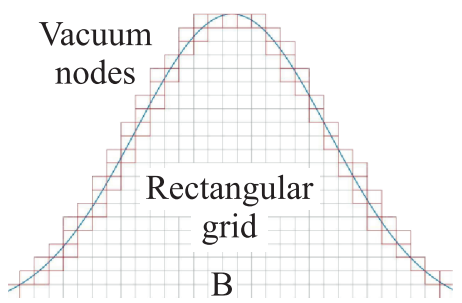

Figure 2. Example of a staircase grid. The grid border tries to repeat the shape of the surface (blue line)

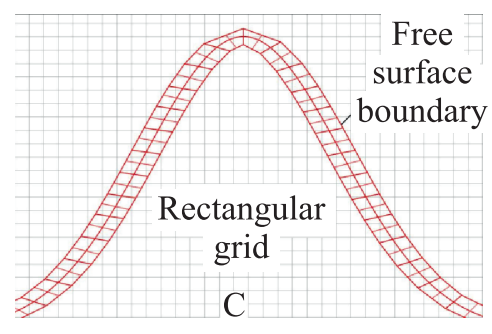

Figure 3. Example of an overset grid. The red lines represent an overset curved grid on the base structured grid (black lines)

\section{Wave equations}

The basic equations of motion of the linear-elastic medium can be written as follows [Aki, Richards, 2002; LeVeque, 2002; Zhdanov, 2002; Zhdanov, 2015]:

$$
\begin{gathered}
\rho \boldsymbol{v}_{t}=(\nabla \cdot \mathbf{T})^{\mathrm{T}}, \\
\mathbf{T}=\lambda(\nabla \cdot \boldsymbol{v}) \mathbf{I}+\mu\left(\nabla \otimes \boldsymbol{v}+(\nabla \otimes \boldsymbol{v})^{\mathrm{T}}\right),
\end{gathered}
$$

where $\rho$ is density, $\boldsymbol{v}$ is velocity, $T$ is the stress tensor, $\lambda$ and $B \mu$ are Lame's parameters characterizing the elastic properties of the medium, $\mathbf{I}$ is the identity tensor of rank, and $\nabla \otimes \boldsymbol{v}$ is a tensor product of two vectors $(\nabla \otimes \boldsymbol{v})_{i, j}=\nabla_{i} \boldsymbol{v}_{j}$.

The system of equations describing the acoustic wave propagation can be written as follows [Landau, Lifshitz, 1959]:

$$
\begin{gathered}
\rho \boldsymbol{v}_{t}=-\nabla p, \\
p_{t}=-c^{2} \rho(\nabla \cdot \boldsymbol{v}),
\end{gathered}
$$


where $\rho$ is an acoustic pressure field in the medium, $\boldsymbol{v}$ is the velocity of acoustic wave propagation, and $c$ is the speed of sound in the acoustic medium. Note that equations (3) and (4) hold for acoustic wave propagation within an incompressible fluid as well.

\section{The grid-characteristic method for numerical modeling of elastic waves in an inhomogeneous medium}

The grid-characteristic method uses the characteristic properties of the systems of hyperbolic equations describing the elastic wave propagation [Petrov et al., 2013; Golubev et al., 2013]. The mathematical principles of the GCM approach are based on representing the equations of motion of the linear-elastic medium in the following form:

$$
\mathbf{q}_{t}+\mathbf{A}_{1} \mathbf{q}_{x}+\mathbf{A}_{2} \mathbf{q}_{y}=0 .
$$

In the last equation, $\boldsymbol{q}$ is a vector of unknown fields, having 5 components and equal to

$$
\mathbf{q}=\left[\begin{array}{c}
\boldsymbol{v} \\
\mathbf{T}
\end{array}\right]=\left[\begin{array}{lllll}
v_{1} & v_{2} & T_{11} & T_{22} & T_{12}
\end{array}\right]^{\mathrm{T}} .
$$

Matrices $\mathbf{A}_{k}, k=1,2$, are the square $5 \mathrm{C} \ldots 5$ matrices. The product of matrix $\mathbf{A}_{k}$ and vector $\boldsymbol{q}$ can be calculated as follows:

$$
\mathbf{A}_{k}\left[\begin{array}{c}
\boldsymbol{v} \\
\mathbf{T}
\end{array}\right]=-\left[\begin{array}{c}
\rho^{-1}(\mathbf{T} \cdot \mathbf{n}) \\
\lambda(\boldsymbol{v} \cdot \mathbf{n}) \mathbf{I}+\mu(\mathbf{n} \otimes \boldsymbol{v}+\boldsymbol{v} \otimes \mathbf{n})
\end{array}\right] .
$$

In the last equation $\boldsymbol{n}$ is a unit vector directed along the $x, y$ directions for matrices $\mathbf{A}_{1}, \mathbf{A}_{2}$, respectively. As we discussed above, the GCM approach is based on representing the solutions of the acoustic and/or elastic wave equations at a later time as a linear combination of the solution displaced at a certain spatial step at some previous instant of time. This representation can be used to construct a direct time-stepping iterative algorithm of computing the wave fields at any instant of time from the initial and boundary conditions. In order to develop this time-stepping formula, we represent matrices $\mathbf{A}_{k}$ using their spectral decomposition. For example, for matrix $\mathbf{A}_{1}$ we have

$$
\mathbf{A}_{1}=\left(\boldsymbol{\Omega}_{1}\right)^{-1} \boldsymbol{\Lambda}_{1} \boldsymbol{\Omega}_{1},
$$

where $\boldsymbol{\Lambda}_{1}$ is a $5 \times 5$ diagonal matrix formed by the eigenvalues of matrix $\mathbf{A}_{1}$; and $\boldsymbol{\Omega}_{1}^{-1}$ is a $5 \times 5$ matrix formed by the corresponding eigenvectors. Note that matrices $\mathbf{A}_{1}$ and $\mathbf{A}_{2}$ have the same set of eigenvalues:

$$
\left\{c_{\mathrm{p}},-c_{\mathrm{p}},-c_{\mathrm{s}}, c_{\mathrm{s}}, 0,0,\right\} .
$$

In the last formula, $\mathrm{C}_{p}$ is a $P$-wave velocity equal to

$$
\left(\rho^{-1}(\lambda+2 \mu)\right)^{0.5}
$$

and $C_{s}$ is an $S$-wave velocity equal to

$$
\left(\rho^{-1} \mu\right)^{0.5} \text {. }
$$

It is shown in [Favorskaya et al., 2018; Khokhlov, Golubev, 2019] that the solution of equation (5), vector $q$, at the $x, y$ and $z$ directions can be written as follows:

$$
\begin{aligned}
& \mathbf{q}(t+\tau, x, y)=\sum_{j=1}^{J} \mathbf{X}_{1, j} \mathbf{q}\left(t, x-\Lambda_{1, j} \tau, y\right), \\
& \mathbf{q}(t+\tau, x, y)=\sum_{j=1}^{J} \mathbf{X}_{2, \mathbf{j}} \mathbf{q}\left(t, x, y-\Lambda_{2, j} \tau\right) .
\end{aligned}
$$


Here $\tau$ is the time step of the solution, $\mathbf{X}_{1, j}$ and $\mathbf{X}_{2, j}$ are the characteristic matrices expressed using the components of matrices $\mathbf{A}_{1}, \mathbf{A}_{2}$ and their eigenvalues as follows:

$$
\mathbf{X}_{\mathrm{i}, j}=\varpi_{* \mathrm{i}, j} \varpi_{i, j}, \quad i=1,2,
$$

where $\varpi_{* \mathrm{i}, \mathrm{j}}$ is the $j$ th column of matrix $\left(\boldsymbol{\Omega}_{\mathrm{i}}\right)^{-1}$, and $\varpi_{\mathrm{i}, \mathrm{j}}$ is the $j$ th row of matrix $\boldsymbol{\Omega}_{\mathrm{i}}$. The scalar components of the column matrices $\varpi_{* i, j}$ are defined by the following expressions:

$$
\begin{gathered}
\omega_{1,2}=\left(\boldsymbol{\Omega}_{1}\left[\begin{array}{c}
\boldsymbol{v} \\
\mathbf{T}
\end{array}\right]\right)_{1,2}=\mathbf{n} \cdot \boldsymbol{v} \mp\left(c_{\mathrm{p}} \rho\right)^{-1}\left(\mathbf{N}_{00} * \mathbf{T}\right), \\
\omega_{3,4}=\mathbf{n}_{1} \cdot \boldsymbol{v} \mp\left(c_{\mathrm{s}} \rho\right)^{-1}\left(\mathbf{N}_{01} * \mathbf{T}\right) \\
\omega_{5,6}=\mathbf{n}_{2} \cdot \boldsymbol{v} \mp\left(c_{\mathrm{s}} \rho\right)^{-1}\left(\mathbf{N}_{02} * \mathbf{T}\right) \\
\omega_{7}=\mathbf{N}_{12} * \mathbf{T} \\
\omega_{8}=\left(\mathbf{N}_{11}-\mathbf{N}_{22}\right) * \mathbf{T} \\
\omega_{9}=\left(\mathbf{N}_{11}+\mathbf{N}_{22}-\frac{2 \lambda}{\lambda+2 \mu} \mathbf{N}_{00}\right) * \mathbf{T}
\end{gathered}
$$

In equations (15)-(20) the asterisk "*" denotes the convolution of two tensors of rank 2 . Expressions (14)-(15) can be used to find the solution, vector $\boldsymbol{q}$, at any time instant, $t+\tau$, from the given initial conditions, thus representing a direct time-stepping algorithm of numerically modeling the elastic wave propagation in inhomogeneous media.

In order to accurately take into account the conditions on the boundary of the modeling domain and on the interfaces inside the modeling domain between the elastic bodies with different properties, we use boundary conditions on the given traction and on a given velocity. Boundary and interface conditions are addressed extensively in [Favorskaya et al., 2018].

\section{The grid-characteristic method for numerically modeling elastic waves in an acoustic medium}

We use the grid-characteristic method for numerically modeling the waves in an acoustic medium as well. In the case of acoustic waves, the vector $\boldsymbol{q}$ of unknown fields has three components, and it is equal to the following expression:

$$
\mathbf{q}=\left[\begin{array}{l}
\boldsymbol{v} \\
p
\end{array}\right]=\left[\begin{array}{lll}
v_{1} & v_{2} & p
\end{array}\right]^{\mathrm{T}}
$$

In the acoustic case, matrices $\mathbf{A}_{k}, k=1,2$ become square $3 \times 3$ matrices. The product of matrix $\mathbf{A}_{k}$ and vector $\boldsymbol{q}$ can be calculated as follows:

$$
\mathbf{A}_{k}\left[\begin{array}{l}
\boldsymbol{v} \\
p
\end{array}\right]=\left[\begin{array}{c}
\rho^{-1} p \mathbf{n} \\
c^{2} \rho(\mathbf{n} \cdot \boldsymbol{v})
\end{array}\right]
$$

In the last equation, $n$ is a unit vector directed along the $x, y$ directions for matrices $\mathbf{A}_{1}, \mathbf{A}_{2}$, respectively. Note that matrices $\mathbf{A}_{1}, \mathbf{A}_{2}$ have the same set of the eigenvalues:

$$
\{c,-c, 0,0\}
$$




\section{Overset grids}

The main idea of this paper is to use the overset mesh approach. Its basis is to cover the wave propagation simulation area by multiple overset grids [Ruzhanskaya, Khokhlov, 2018], allowing more precise results to be achieved when solving problems which require grids to be codirectional with some problem geometry axes and when more rough methods fail to provide acceptable results. Despite the fact that reducing spatial and temporal steps can increase the accuracy of calculations, in the case of a problem statement involving multiple objects, each with its own direction, quadrilateral grids may be unable to fulfill the accuracy requirements. The proposed approach is not innovative, and several works analyzing its workability in combination with different methods exist.

Figure 4 represents a half-space, filled with soil, where elastic wave propagates, with a semicircle cut, filled with air, representing a canyon. Figure 5 shows one of the grid layout options.

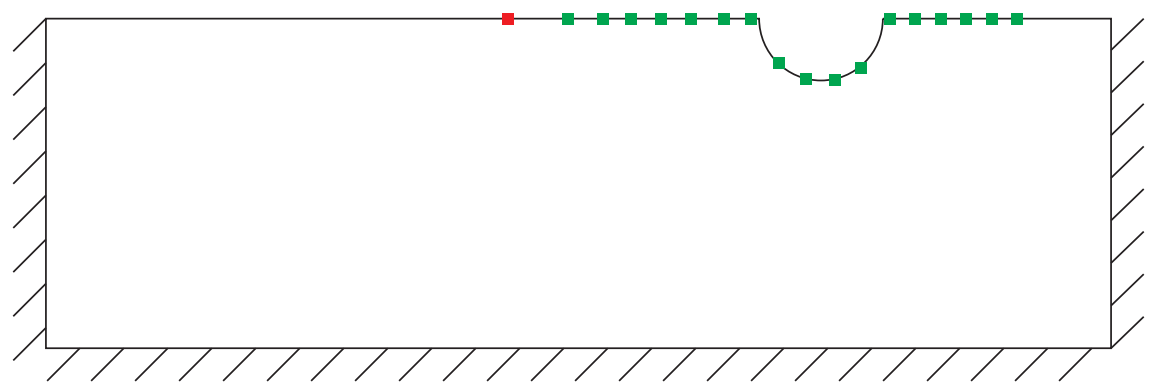

Figure 4. Illustration of the statement of the Canyon problem. The red square indicates the position of the wave source, the green squares indicate the positions of the receiver

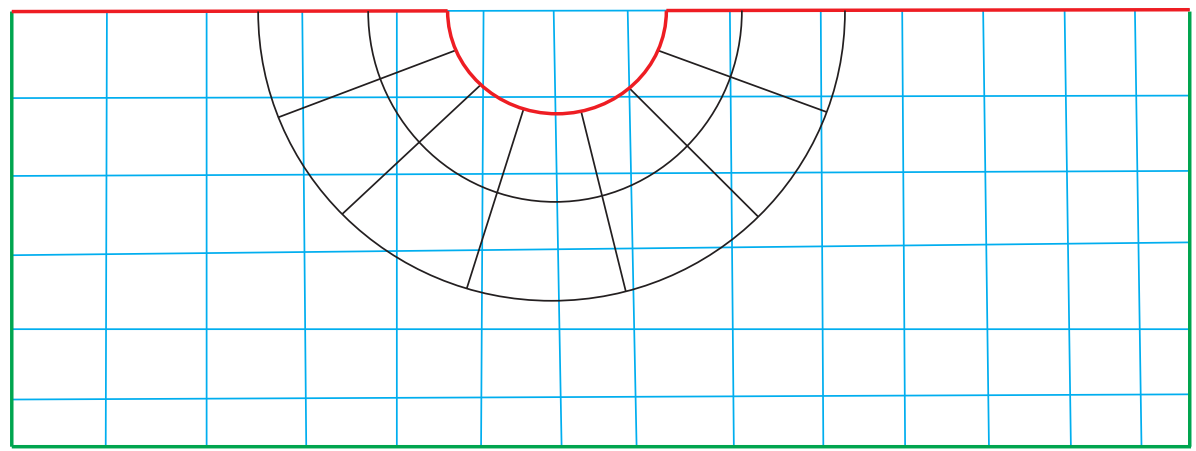

Figure 5. Layout scheme of the Canyon problem grid. The blue lines represent a regular rectangular grid, the black lines represent a curvilinear grid following the canyon surface. The boundaries with free surface boundary conditions are illustrated by red lines, the ones with absolute absorbing boundary conditions are illustrated by green lines

Grid interpolation only happens at the boundary of the black (curvilinear) grid, all blue grid nodes located in the area covered by the black grid have their values overwritten, based on the values of the black grid node, so that wave propagation in this area is completely simulated using the black grid, and the results of this simulation are copied to the blue grid taking into account their influence on the other part of the area, and force the probably incorrect values in the blue grid, simulated without free surface condition on the canyon boundary, to be erased.

In problem statements and models that require multiple grids, the process of simulation is independent for each grid, and thus can be processed in parallel. The implementation of GCM with parallel grid processing was described in [Ivanov, Khokhlov, 2019]. 
The term interpolation is used in this abstract to describe the process of ascertainment of values of the function in the nodes of one of the grids using its values in the nodes of the other grid. In the case of overset grids, interpolation is used to provide correctness of modeling interactions described by different grids, and taking their contributions to the final result into account. If the area described by one of our grids is part of an area described by the other grid (this is the case in our study), interpolation allows specifying the solution in the area, described by the smaller (and more precise) grid. In this case, it makes sense to overwrite the values in the larger grid nodes and replace them with those received from the interpolation process, as they are more precise. As for interpolating from the larger grid to the smaller one, it is sufficient to overwrite only the values in the nodes near the boundary of the smaller grid, because it both allows us to keep the values of the smaller grid's node correct (and take the interaction into account), and avoid the unnecessary process of re-interpolating them from the larger grid nodes, which were previously interpolated from them (and this process can probably introduce some additional error).

It should be noted that the process of interpolation introduces an error due to possible differences between the values of the real function, and those built by values in nodes during the interpolation.

In this paper, bilinear interpolation is used. This type of interpolation is one of the simplest and the fastest. In each cell of the grid, the interpolated function is approximated by the following:

$$
f(x, y)=A+B x+C y+D x y
$$

with coefficients $A, B, C$ and $D$ calculated using the node coordinates and function values. Usually, bilinear interpolation is used when the nodes in which the function values are known are located in some rectangle corners, but in this article it was generalized for the case of a nondegenerate convex quadrilateral. Future plans involve analyzing the expediency of using other interpolation methods including, but not limited to, bicubic interpolation and spline interpolation. Analyzing the expediency of conservative interpolation usage is planned as well.

\section{Results}

Method validation was performed by comparison with [Favorskaya et al., 2018] in the case of two-dimensional Lamb's problem solving. As you can see in Fig. 6 and Fig. 7, all three seismograms have deviations of the same order; as a result, we have decided that our method is satisfactory.

To demonstrate the capabilities of our method, we calculate the propagation of the seismic wave in the solid for the Canyon problem (Fig. 8). Base (regular rectal) grid dimensions equal 900 and 450 cells (width and height, respectively). Curved grid dimensions equal 100 and 10 cells (width and height respectively). The time step is 0.001 seconds. The physical solid characteristics are $\rho=1000$, $V_{p}=2000, V_{s}=1000$. To validate the result of our approach in the case of nontrivial topography, we compare it with "specfem $2 \mathrm{~d}$ " solution. We used the 4th-order 6-stage low storage Runge-Kutta scheme, a nonregular rectangle grid with the same grid dimensions and time step [Wang, Cai, 2016].

Taking into account the topography of the Earth's surface can improve the accuracy of seismic modeling when using real geological data. The presence of topography will cause more complicated seismic wave propagation phenomena, such as diffraction at rough surfaces, complex propagation of Rayleigh waves, and side effects caused by wave interferences. The primary goal of this study is to construct a method that implements the free surface on topography, utilizing an overlay curved grid for characterization. We investigate the workability of the GCM approach using overset grids (also known as the Chimera grid approach) in a combination of regular rectangular and curvilinear grids. Furthermore, this mechanism allows computational complexity to be reduced by simulating wave propagation in the regular, homogeneous physical area using a sparse regular rectangle grid, in order to increase the digitalization of fractured regions and minimize the Courant number. Moreover, 
Seismogram comparison for free surface reflection
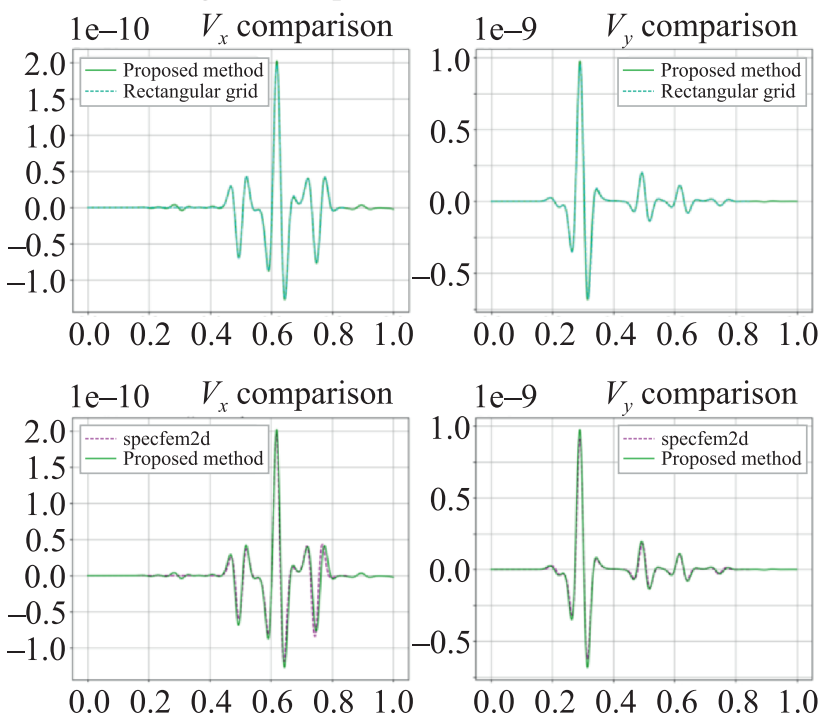

Figure 6. Pairwise comparison of various solutions

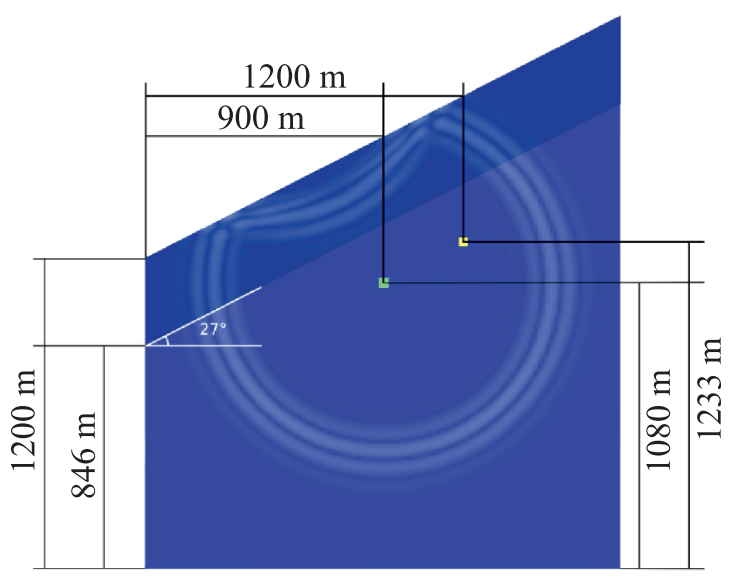

Figure 7. Problem statement with the applied overset grid. The light blue region represents a structured regular grid and dark blue is the overset curved grid. The source and receiver positions are indicated by green and yellow squares, respectively

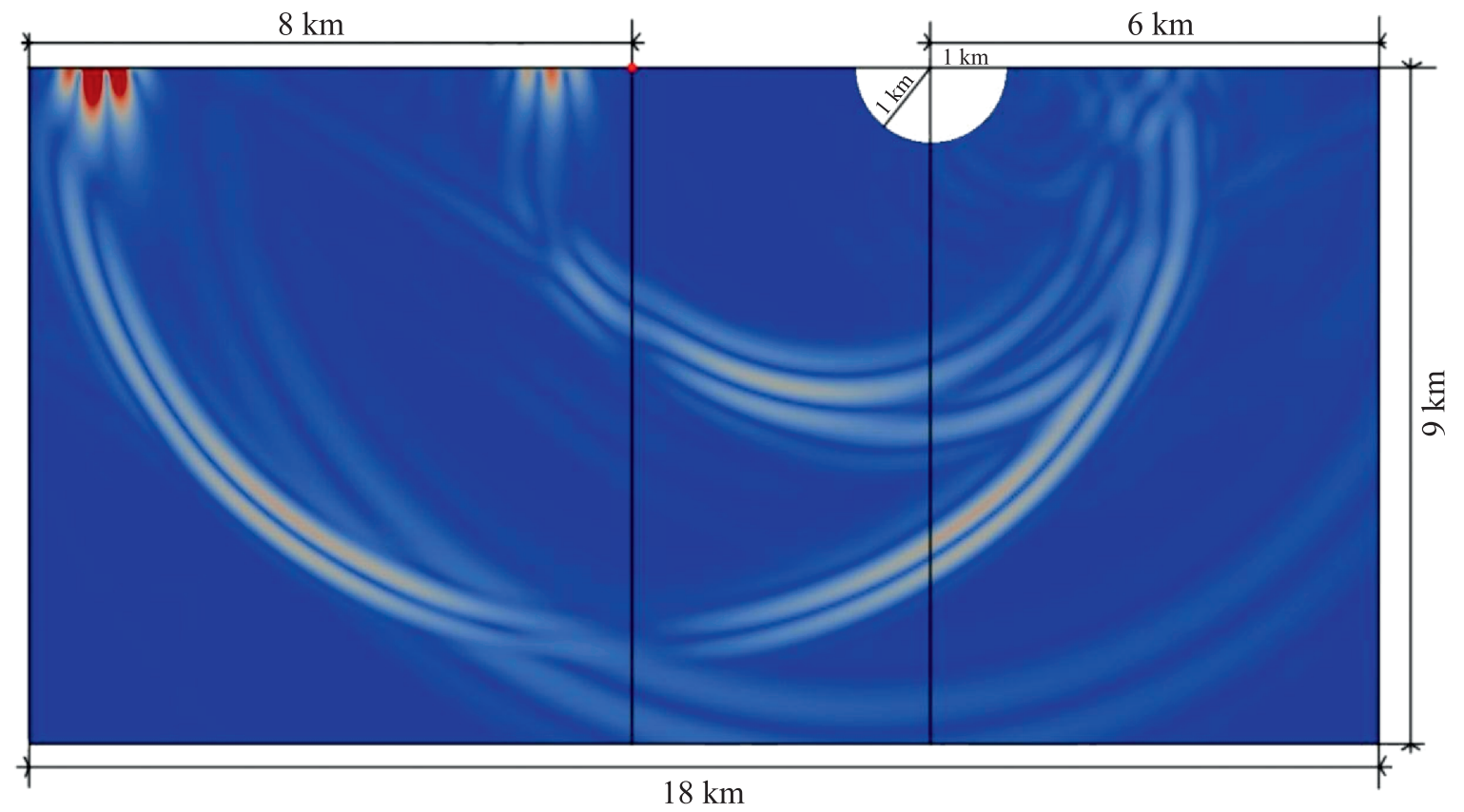

Figure 8. Illustration of the statement of the Canyon problem. The red square indicates the position of the wave source, the green squares indicate receiver positions

the mechanism of mesh building is simplified. Method validation was performed by comparison with "specfem $2 \mathrm{~d}$ " software packages in solving the two-dimensional Lamb problem. We used the 4th-order 6-stage low storage Runge-Kutta scheme, a nonregular rectangle grid with the same grid dimensions and time step. To demonstrate the capabilities of our method, we calculate the propagation of the seismic wave in the solid under wavy free-surface. To validate the results of our approach in the case of nontrivial topography, we compare it with "specfem $2 \mathrm{~d}$ " solution in the "canyon" problem. 

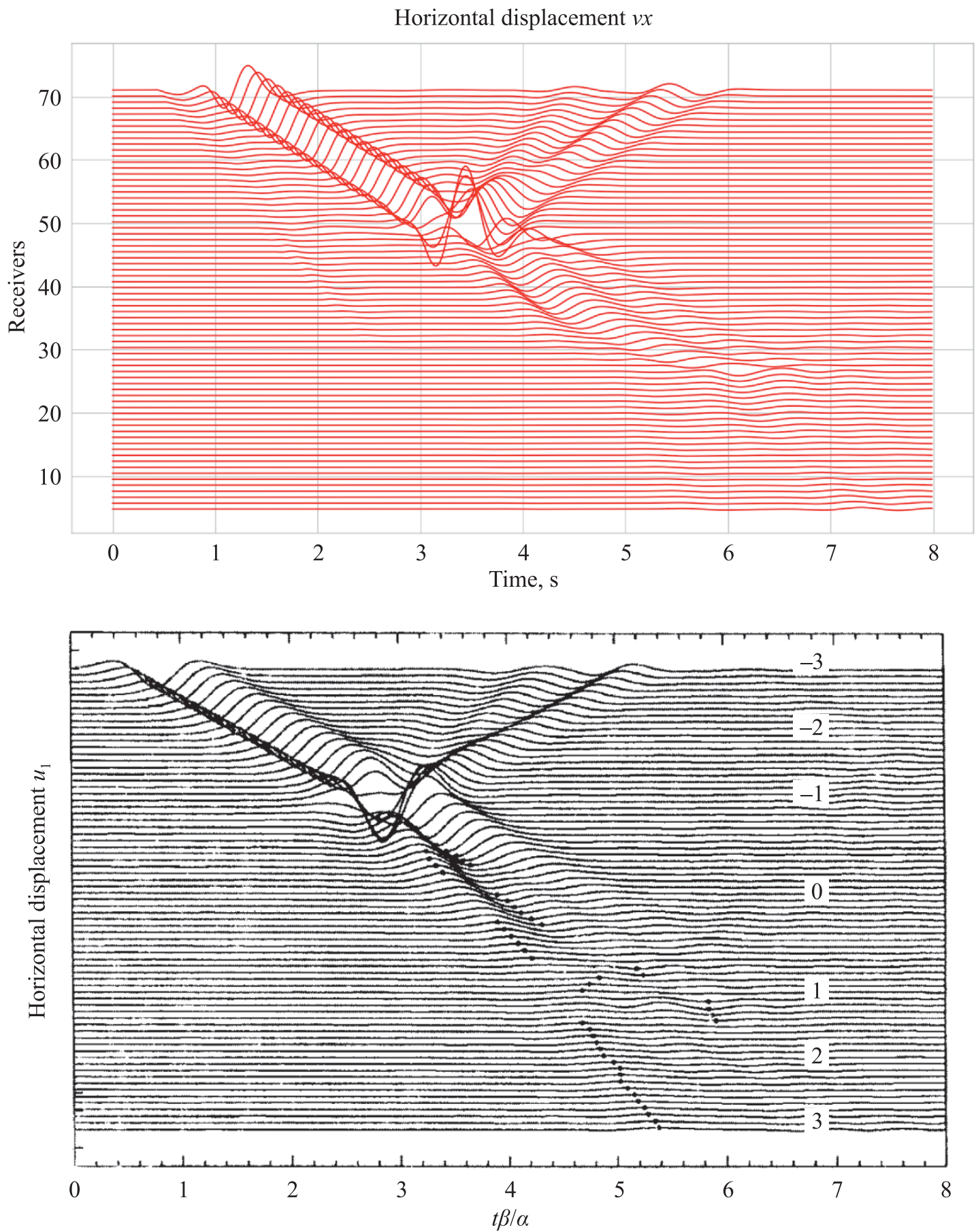

Figure 9. Canyon problem statement illustration. The red square indicates wave source position, green squares indicate receiver positions

We have presented a novel approach for modeling of surface topography. This approach significantly reduces the computation time due to more simple processing of computational grids. In this study all models were assumed to be $2 \mathrm{D}$. However, extension of the approach to a $3 \mathrm{D}$ case is straightforward. This will be a topic of further research. 


\section{References}

Aki K., Richards P. G. Quantitative seismology, theory and methods. - Second edition, University Science Books, Sausalito, California, 2002.

Biryukov V.A., Miryakha V.A., Petrov I. B., Khokhlov N.I. Simulation of elastic wave propagation in geological media: Intercomparison of three numerical methods // Computational Mathematics and Mathematical Physics. - 2016. - Vol. 56(6). - P. 1086-1095.

Cao J., Chen J.-B. A parameter-modified method for implementing surface topography in elastic-wave finite-difference modeling // Geophysics. - 2018. - Vol. 83(6). - P. T313-T332.

Cruz-Atienza V.M., Virieux J. Dynamic rupture simulation of non-planar faults with a finite-difference approach // Geophysical Journal International. - 2004. - Vol. 158(3). - P. 939-954.

Dortdivanlioglu B., Krischok A., Beirćo da Veiga L., Linder C. Mixed isogeometric analysis of strongly coupled diffusion in porous materials // International Journal for Numerical Methods in Engineering. - 2018. - Vol. 114(1). - P. 28-46.

Etienne V., Chaljub E., Virieux J., Glinsky N. An hp-adaptive discontinuous Galerkin finiteelement method for 3-D elastic wave modeling // Geophysical Journal International. - 2010. Vol. 183(2). - P. 941-962.

Favorskaya A.V., Zhdanov M.S., Khokhlov N.I., Petrov I.B. Modeling the wave phenomena in acoustic and elastic media with sharp variations of physical properties using the grid-characteristic method // Geophysical Prospecting. - 2018. - Vol. 66(8). - P. 1485-1502.

Gao L., Brossier R., Pajot B., Tago J., Virieux J. An immersed free-surface boundary treatment for seismic wave simulation // Geophysics. - 2015. - Vol. 80(5). - P. T193-T209.

Golubev V.I., Petrov I. B., Khokhlov N.I. Numerical simulation of seismic activity by the gridcharacteristic method // Computational Mathematics and Mathematical Physics. - 2013. Vol. 53(10). - P. 1523-1533.

Gottschammer E. Accuracy of the Explicit Planar Free-Surface Boundary Condition Implemented in a Fourth-Order Staggered-Grid Velocity-Stress Finite-Difference Scheme // Bulletin of the Seismological Society of America. - 2001. - Vol. 91(3). - P. 617-623.

Graves R.W. Simulating Seismic Wave Propagation in 3D Elastic Media Using Staggered-Grid Finite Differences // Bulletin of the Seismological Society of America. - 1996. - Vol. 86(4). - P. 10911106.

Ivanov A.M., Khokhlov N.I. Efficient Inter-process Communication in Parallel Implementation of Grid-Characteristic Method // Smart Modeling for Engineering Systems. - 2019. - P. 91-102. — Springer International Publishing.

Jianfeng Z. Quadrangle-grid velocity-stress finite-difference method for elastic-wave-propagation simulation // Geophysical Journal International. - 1997. - Vol. 131(1). - P. 127-134.

Khokhlov N.I., Golubev V.I. On the Class of Compact Grid-Characteristic Schemes // Smart Modeling for Engineering Systems. - 2019. - P. 64-77. - Springer International Publishing.

Komatitsch D., Tromp J. Introduction to the spectral element method for three-dimensional seismic wave propagation // Geophysical Journal International. - 1999. - Vol. 139(3). - P. 806-822.

Kristek J., Moczo P., Archuleta R. J. Studia Geophysica et Geodaetica. - 2002. - Vol. 46(2). - P. 355381.

Landau L.D., Lifshitz E. M. Fluid mechanics. - Pergamon Press, 1959.

LeVeque R. Finite volume methods for hyperbolic problems. - Cambridge University Press, 2002.

Liu S., Li X., Wang W., Liu Y. A mixed-grid finite element method with PML absorbing boundary conditions for seismic wave modeling // Journal of Geophysics and Engineering. - 2014. Vol. 11(5). - P. 55009. 
Ma S. Hybrid Modeling of Elastic P-SV Wave Motion: A Combined Finite-Element and StaggeredGrid Finite-Difference Approach // Bulletin of the Seismological Society of America. - 2004 . Vol. 94(4). - P. 1557-1563.

Meng $W$., Fu L.-Y. Seismic wavefield simulation by a modified finite element method with a perfectly matched layer absorbing boundary // Journal of Geophysics and Engineering. - 2017. Vol. 14(4). - P. 852-864.

Peng K., Rao Y., Wang Y. Micro-seismic Wave Simulation with the Effect of Pore Pressure During Hydraulic Fracturing // In 80th EAGE Conference and Exhibition 2018. - 2018. - EAGE Publications BV.

Pérez S. C. A., Donno D., Chauris H. (2016). Finite-difference strategy for elastic wave modeling on curved staggered grids. Computational Geosciences, 20(1), 245-264.

Peter D., Komatitsch D., Luo Y., Martin R., Le Goff N., Casarotti E., ..., Tromp J. Forward and adjoint simulations of seismic wave propagation on fully unstructured hexahedral meshes // Geophysical Journal International. - 2011. - Vol. 186(2). - P. 721-739.

Petersson N.A., Sjögreen B. Wave propagation in anisotropic elastic materials and curvilinear coordinates using a summation-by-parts finite difference method // Journal of Computational Physics. - 2015. - Vol. 299. - P. 820-841.

Petrov I.B., Favorskaya A. V., Sannikov A.V., Kvasov I. E. Grid-Characteristic Method Using High Order Interpolation on Tetrahedral Hierarchical Meshes with a Multiple Time Step // Mathematical Models and Computer Simulations. - 2013. - Vol. 5(5). - P. 409-415.

Petrov I. B., Favorskaya A.V., Khokhlov N.I. Grid-characteristic method on embedded hierarchical grids and its application in the study of seismic waves // Computational Mathematics and Mathematical Physics. - 2017. - Vol. 57(11). - P. 1771-1777.

Ruzhanskaya A., Khokhlov N. Modeling of Fractures Using the Chimera Grid Approach // In 2nd Conference on Geophysics for Mineral Exploration and Mining. - 2018. - EAGE Publications BV.

Saenger E.H., Gold N., Shapiro S.A. Modeling the propagation of elastic waves using a modified finite-difference grid // Wave Motion. - 2000. - Vol. 31(1). - P. 77-92.

Stognii P. V., Khokhlov N.I. 2D Seismic Prospecting of Gas Pockets // In Smart Modeling for Engineering Systems. - 2019. - P. 156-166. - Springer International Publishing.

Tarrass I., Giraud L., Thore P. New curvilinear scheme for elastic wave propagation in presence of curved topography // Geophysical Prospecting. - 2011. - Vol. 59(5). - P. 889-906.

Virieux J., Calandra H., Plessix R.-É. A review of the spectral, pseudo-spectral, finite-difference and finite-element modeling techniques for geophysical imaging // Geophysical Prospecting. - 2011. Vol. 59(5). - P. 794-813.

Wang X., Cai M. FLAC/SPECFEM2D coupled numerical simulation of wavefields near excavation boundaries in underground mines // Computers \& Geosciences. - 2016. - Vol. 96. - P. 147-158.

Zeng C., Xia J., Miller R.D., Tsoflias G.P. An improved vacuum formulation for 2D finitedifference modeling of Rayleigh waves including surface topography and internal discontinuities // Geophysics. - 2012a. - Vol. 77(1). - P. T1-T9.

Zhang $W$., Zhang Z., Chen $X$. Three-dimensional elastic wave numerical modeling in the presence of surface topography by a collocated-grid finite-difference method on curvilinear grids // Geophysical Journal International. - 2012b. - Vol. 190(1). - P. 358-378.

Zhdanov M. S. Geophysical Inverse Theory and Regularization Problems. - Elsevier, 2002.

Zhdanov M. S. Inverse Theory and Applications in Geophysics. - Elsevier, 2015. 
